\title{
Características de cepillado y lijado de 33 especies de madera
}

\author{
Enrique Martínez-Pinillos Cueto ${ }^{1}$ \\ José Luis Martínez Castillo ${ }^{1}$
}

\begin{abstract}
RESUMEN
Existe un número de especies de madera sobre las que aún no se conoce su respuesta a diferentes operaciones de maquinado; esto propicia que su uso sea limitado aún teniendo un gran potencial comercial. Por este motivo es útil la realización de estudios para conocer la respuesta que cada especie tiene frente a diferentes procesos de maquinado. Se probaron 33 especies de madera de diferentes regiones del país. Este documento complementa un primer trabajo en el cuál, se presentan los resultados de los procesos de barrenado, escopleado y moldurado para las mismas especies. La metodología usada es la establecida por la norma ASTM D-1666-64 (American Society for Testing and Materials), y los procesos probados fueron cepillado y lijado. En la prueba de cepillado se usaron tres diferentes ángulos de corte en las cuchillas, $20^{\circ}, 25^{\circ}$ y $30^{\circ}$. Para la prueba de lijado se usaron lijas con tres diferentes granulaciones 60,80 y 100 . Los resultados se muestran en grupos de especies de acuerdo con la calidad de la superficie maquinada; esta información puede ser útil para personas que fabrican productos usando madera pues indica la calidad que cada una de las especies probadas tendrá al ser sometida a cualquiera de los procesos estudiados.
\end{abstract}

PALABRAS CLAVE:

Maquinado de madera, cepillado, lijado, ángulo de corte, graduaciones de lijas.

\section{ABSTRACT}

The lack of knowledge about wood machining properties may be one of the reasons that potentially commercial wood is not utilized. For this reason it is necessary to perform wood machining tests. This paper presents the results of planing and sanding tests carried out on thirty-three wood species from different regions of Mexico. In a previous study, three types of tests were carried out: boring, mortising and shaping. The ASTM D-1666-64 (American Society for Testing and Materials) Standard methodology was followed. For planing, three different knife angles were used: $20^{\circ}$, $25^{\circ}$ and $30^{\circ}$. For sanding tests, three kinds of sandpapers were used: 60,80 and 100 grade. Results were grouped according to the surface quality obtained. This information could be useful for those involved in manufacturing wood products since it indicates the surface quality for each of the species tested when subjected to the above mentioned machining processes.

KEY WORDS:

Planing, sanding, wood machinig, angle knife, sandpaper grades.

1 Técnicos Académicos. Departamento de Productos Forestales y Conservación de Bosques. Instituto de Ecología, A.C. Apdo. Postal 63. 91000 Xalapa, Ver. México. Manuscrito recibido para su publicación el 23 de Noviembre de 1994 


\section{INTRODUCCION}

Un aspecto muy importante que es necesario conocer y tomar en cuenta cuando se desea transformar a la madera en productos es la respuesta que esta materia prima tendrá cuando se someta a las operaciones de maquinado que el proceso requiera. Este trabajo muestra los resultados que las especies de maderas seleccionadas presentaron cuando fueron sometidas a las operaciones de cepillado y lijado. Esta información es muy útil para quienes usan este tipo de especies y quieren mejorar sus procesos o para quien desea empezar a usar alguna de las especies de madera estudiadas. Con esta información también se contribuye a promocionar el uso de maderas poco utilizadas, pero que tienen un alto potencial comercial y de esta manera bajar la presión sobre algunas especies para contribuir a un uso más racional de los recursos maderables. Las operaciones que pueden quedar involucradas en un proceso de transformación de la madera son varias, pero en este trabajo solo se tocan las operaciones de cepillado y lijado.

Estas operaciones tienen gran importancia en cualquier proceso de producción, pues es muy raro que no aparezcan en alguna línea de productos de artículos de madera. El cepillado, en general, es el primer proceso a que se somete la madera, ya que con este proceso se obtiene un grosor uniforme en las piezas que se utilizarán en los procesos posteriores, con lo cual se reducen muchos problemas de fabricación posteriores, incrementándose con esto la calidad del producto por fabricar. El lijado forma parte de la fase final en la fabricación de los productos, su función es obtener en la madera superficies con características aceptables para poder aplicar el acabado final.

\section{OBJETIVO}

Determinar la calidad del acabado obtenido por los procesos de cepillado y lijado, en cada una de las especies de madera sometidas a estas operaciones de maquinado.

\section{METODOLOGIA GENERAL}

Para la realización de este trabajo se utilizaron 33 especies de madera con densidades que varían desde 0.19 hasta 0.90 , este material fue seleccionado, dimensionado y secado. Todo el material se colocó en un cuarto con temperatura y humedad relativa controladas hasta lograr un contenido de humedad en equilibrio de $12 \%$ para todas las probetas como lo establece la Norma ASTM D-1666 (ASTM, 1993); se usaron cinco probetas por especie. Para los dos tipos de prueba se utilizó la misma probeta con dimensiones de $90 \times 12.5 \mathrm{~cm}, 22 \mathrm{~mm}$ de grueso y se cortó de una pieza de $120 \times 12.5 \mathrm{~cm}$, como se observa en la figura 1.

A cada una de las especies estudiadas se les determinó su valor de densidad relativa peso anhidro/volumen verde (pa/vv). Con este valor se procedió a ordenar las especies en forma ascendente (Tabla 1). De acuedo con este orden se procedió a efectuar las pruebas, empezando por la de más baja densidad y finalizando con la más alta. Esto fue con el fin de disminuir al máximo el desgaste en el filo de las herramientas de corte.

\section{Equipo}

De acuerdo con las especificaciones de cada máquina y las de la Norma ASTM D-1666 (1984), se procedió a probar y calibrar la maquinaria que se usaría, sometiéndola también, a una rutina de mantenimiento adecuada, dejando así el equipo en perfectas condiciones para iniciar las pruebas. Se obtuvieron las 
Tabla 1. Especies ensayadas en los procesos de cepillado y lijado

\begin{tabular}{|c|c|c|c|}
\hline ESPECIE & NOMBRE COMUN & $\begin{array}{c}\text { ESTADO } \\
\text { DE COLECTA }\end{array}$ & $\begin{array}{l}\text { DR } \\
\text { (pa/ } \\
\text { vv) }\end{array}$ \\
\hline $\begin{array}{ll}1 & \text { Ceiba pentandra (L.) Gaertm. } \\
2 & \text { Pseudobombax ellipticum (Kunth) Dugand } \\
3 & \text { Bursera simarouba (L.) Sarg. } \\
4 & \text { Cedrela odorata L. } \\
5 & \text { Talauma mexicana (OC.) Dom. } \\
6 & \text { Schizolobium parahybum (Vell.) Blake } \\
7 & \text { Pinus pseudostrobus Lindl. } \\
8 & \text { Persea americana Miller } \\
9 & \text { Acer saccharum Marsh subs. skutchii } \\
& \text { (Rehder) E. Murray } \\
10 & \text { Casuarina cunninghamiana Miq. } \\
11 & \text { Swietenia macrophylla G. King. } \\
12 & \text { Vochysia guatemalensis Donnell Smith } \\
13 & \text { Liquidambar macrophylla Oersted } \\
14 & \text { Lysiloma acapulcensis (Kunth) benth. } \\
15 & \text { Pinus teocote Schlechtendal \& Cham. } \\
16 & \text { Pinus patula Schlechtendal \& Cham. } \\
17 & \text { Guarea grandifolia A. DC. } \\
18 & \text { Vatairea lundellii (Standley) Killip. ex Record } \\
19 & \text { Magnolia schideana Schlechtendal } \\
20 & \text { Fraxinus udhei (Wenzing) Lingelsheim } \\
21 & \text { Quercus sartorii Liebm. } \\
22 & \text { Cupania sp. } \\
23 & \text { Licania platypus (Helms.) Fristch. } \\
24 & \text { Astronium graveolens Jacquim } \\
25 & \text { Lysiloma bahamensis Benth. } \\
26 & \text { Ampelocera hottlei (Standley) Standley } \\
27 & \text { Mirandaceltis monoica } \\
28 & \text { Quercus crassifolia Humb. \& Bonpl. } \\
29 & \text { Lonchocarpus castilloi Standley } \\
30 & \text { Lucuma salicifolia H.B.K. } \\
31 & \text { Dialium guianense (Aubl.) Sandwith } \\
32 & \text { Platymiscium yucatanum Standley } \\
33 & \text { Manilkara zapota (L.) van Royen } \\
& \end{array}$ & $\begin{array}{l}\text { ceiba } \\
\text { amapola } \\
\text { palo mulato } \\
\text { cedro } \\
\text { jolmashté } \\
\text { falso guanacaste } \\
\text { pino } \\
\text { aguacate } \\
\text { álamo } \\
\text { casuarina } \\
\text { caoba } \\
\text { maca blanca } \\
\text { liquidámbar } \\
\text { tripal } \\
\text { pino } \\
\text { pino } \\
\text { cedrillo } \\
\text { amargoso } \\
\text { magnolia } \\
\text { fresno } \\
\text { encino escobillo } \\
\text { casalcahuite } \\
\text { cabeza de mico } \\
\text { jobillo } \\
\text { tzalam } \\
\text { cuerillo } \\
\text { chicharra } \\
\text { encino rojo } \\
\text { machiche } \\
\text { zapote amarillo } \\
\text { paque } \\
\text { hormiguillo } \\
\text { chicozapote }\end{array}$ & $\begin{array}{l}\text { Chiapas } \\
\text { Chiapas } \\
\text { Chiapas } \\
\text { Chiapas } \\
\text { Chiapas } \\
\text { Chiapas } \\
\text { Oaxaca } \\
\text { Veracruz } \\
\text { Tamaulipas } \\
\text { Veracruz } \\
\text { Chiapas } \\
\text { Chiapas } \\
\text { Tamaulipas } \\
\text { Chiapas } \\
\text { Oaxaca } \\
\text { Oaxaca } \\
\text { Chiapas } \\
\text { Chiapas } \\
\text { Tamaulipas } \\
\text { Veracruz } \\
\text { Tamaulipas } \\
\text { Q. Roo } \\
\text { Chiapas } \\
\text { Chiapas } \\
\text { Q. Roo } \\
\text { Chiapas } \\
\text { Chiapas } \\
\text { Veracruz } \\
\text { Chiapas } \\
\text { Q. Roo } \\
\text { Chiapas } \\
\text { Chiapas } \\
\text { Chiapas }\end{array}$ & $\begin{array}{l}0.19 \\
0.24 \\
0.30 \\
0.33 \\
0.34 \\
0.35 \\
0.35 \\
0.40 \\
0.40 \\
0.42 \\
0.42 \\
0.44 \\
0.48 \\
0.49 \\
0.49 \\
0.51 \\
0.51 \\
0.51 \\
0.52 \\
0.53 \\
0.53 \\
0.59 \\
0.61 \\
0.61 \\
0.62 \\
0.64 \\
0.65 \\
0.68 \\
0.71 \\
0.71 \\
0.73 \\
0.73 \\
0.90\end{array}$ \\
\hline
\end{tabular}

herramientas de corte (cuchillas) y abrasivos (lijas) necesarias para efectuar las pruebas de cepillado y lijado. En el caso de las cuchillas para la prueba de cepillado fue necesario afilarlas con los ángulos de corte requeridos. Al finalizar el calibrado de la maquinaria y mantenimiento, se inició con las pruebas.

\section{Proceso de cepillado}

Cepillar o sacar una cara en limpio de la madera es un proceso básico en la transformación de la madera. Esta operación se efectúa en las primeras etapas de la línea de producción de los productos y permite obtener un grosor igual en las pieza y una superficie 


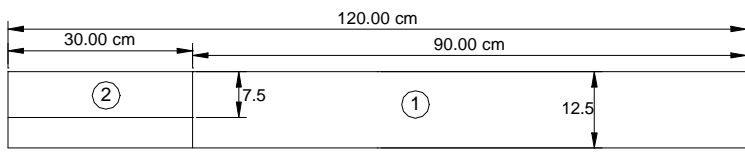

Figura 1. Patrón de corte de los especímenes de prueba

uniforme en las caras. Esta operación se realiza en una máquina denominada cepillo (Fig. 2). El desbaste del material se efectúa con cuchillas que son colocadas en un cabezal que gira mientras la madera es arrastrada en la mesa del cepillo mediante unos rodillos (Fig. 3).

Las variables que rigen el proceso de cepillado de la madera son; las revoluciones por minuto del cabezal, número de cuchillas, velocidad de alimentación de la madera, afilado y ángulo de corte de las cuchillas. En la mayoría de los cepillos el único factor que se puede variar, dentro de los límites que la máquina lo permita, es la velocidad de avance del material, pues las revoluciones por minuto en el cabezal son fijas, así como el ángulo para colocar las cuchillas. Este último factor sí es posible modificarlo con la variación del ángulo de la punta de las cuchillas (Fig. 4), logrando con esto poder tener diferentes ángulos de corte, los que dependiendo de la especie que se desea cepillar podrán ser más 0 menos eficientes.

En el proceso de cepillado cada cuchilla deja una pequeña marca en la superficie de la madera, las que en conjunto y observando con detalle la cara cepillada se notan

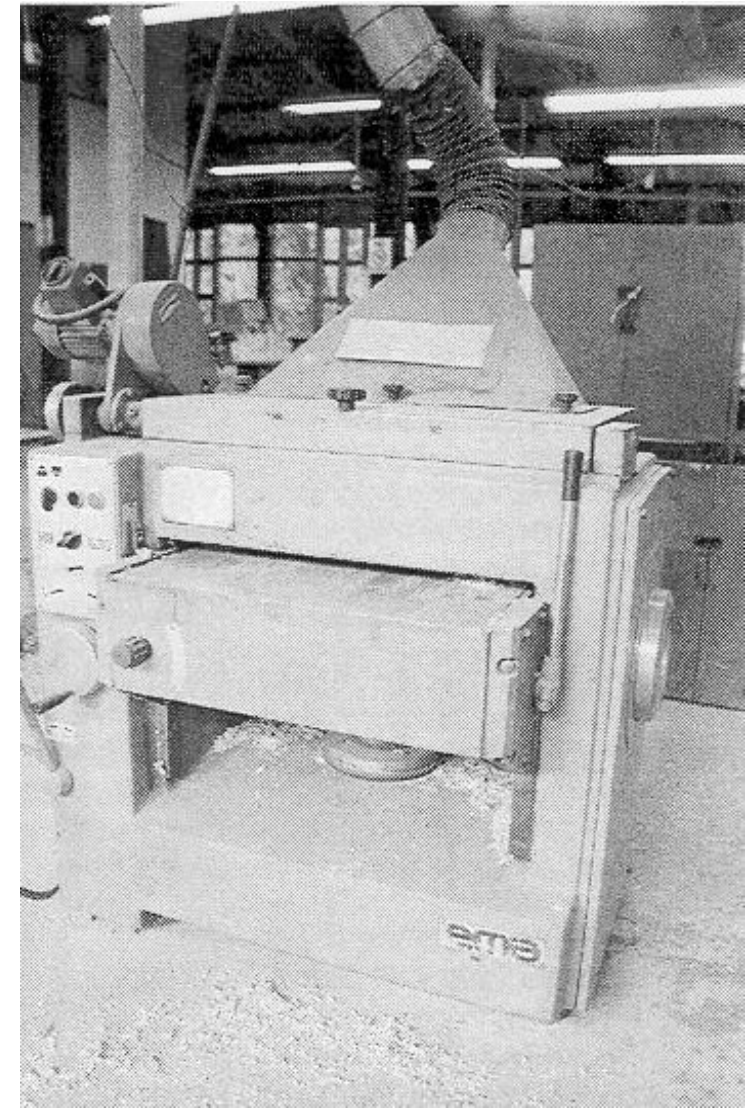

Figura 2. Máquina cepilladora usada en las pruebas

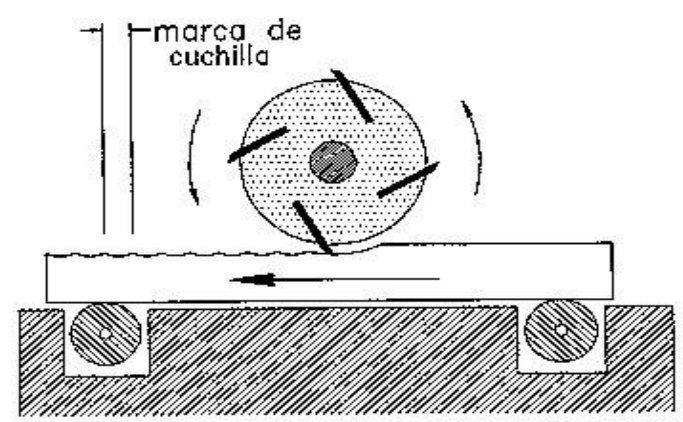

Figura 3. Esquema de corte de las cuchillas en el cepillo 
como una ondulaciones (Fig. 3). La cantidad de estas marcas por pulgada está relacionada con el número de cuchillas, revoluciones por minuto y velocidad de avance (Cantin, 1965). Se puede saber la cantidad de estas marcas por pulgada utilizando la siguiente fórmula:

$$
\text { No. de marcas por pulgada }=\frac{A \times B}{C \times 12}
$$

donde:
$A=$ revoluciones por minuto
$\mathrm{B}=$ número de cuchillas
$C=$ velocidad de avance (pies/minuto)

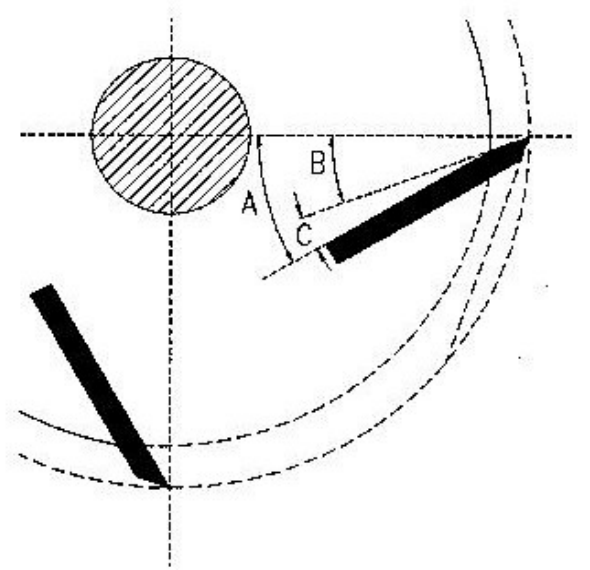

Figura 4. Modificación de los ángulos de corte de las cuchillas $\mathrm{A}=$ ángulo de corte, $\mathrm{B}$ = nuevo ángulo de corte, $\mathrm{C}=$ ángulo de afilado

Para esta prueba se decidió usar tres juegos diferentes de cuchillas; a dos de ellas se les modificó el ángulo de la punta para probar las especies con un ángulo de corte de $20^{\circ}$ y con otro de $25^{\circ}$. El tercer juego se utilizó tal y como se obtiene del distribuidor, con un ángulo de $30^{\circ}$.
La máquina cepilladora que se utilizó fue sometida a una etapa de mantenimiento. En el momento que se encontraba en condiciones aceptables, se procedió a montar el primer juego de cuchillas para realizar las primeras pruebas. El cepillo que se usó tiene en el cabezal una potencia de $12 \mathrm{hp}$ (caballos de fuerza) y espacio para colocar cuatro cuchillas. La potencia del motor de los rodillos de arrastre es de $2.5 \mathrm{hp}$ y una velocidad de avance variable de 20 a 60 pies/minuto. Cuando todo el equipo se encontraba listo, se procedió a probar las especies con el primer ángulo de corte seleccionado.

Cada pieza se transportó del cuarto con humedad y temperatura controladas al taller de carpintería en bolsas de polietileno para disminuir variaciones del contenido de humedad. Se inició con las especies de menor densidad para tratar de minimizar el desafilado de las cuchillas. El cepillo se calibró para desbastar $1.5 \mathrm{~mm}$ de material a cada probeta, todas las probetas fueron cepilladas a favor del grano y al finalizar el cepillado de cada probeta se marcó la cara cepillada y la dirección en que se efectuó el corte para posteriormente evaluar todas las especies usando como criterios de evaluación cinco grados que son: EXCELENTE (1), BUENO (2), REGULAR (3), POBRE (4), MUY POBRE (5); los defectos que se calificaron fueron grano algodonoso, grano arrancado, grano levantado y marcas de viruta asignándole un valor entre 1 y 5 a cada defecto calificado (Longwood, 1961). Todos estos datos se anotaron en una hoja de resultados diseñada para este fin. Al finalizar todo este procedimiento, con un ángulo de corte, se procedió a colocar un nuevo juego de cuchillas con otro ángulo en la punta para realizar las pruebas con las mismas probetas, repitiendo el mismo procedimiento seguido con el ángulo de corte anterior. 


\section{Proceso de lijado}

Esta operación tiene como finalidad corregir los defectos del proceso de cepillado, como son las marcas de viruta, huellas de cuchillas y posibles imperfecciones manifestadas como grano algodonoso, grano arrancado, o grano levantado, o cualquier otro defecto causado por otra operación en la madera. También, representa una de las últimas etapas en el proceso de maquinado de la superficie de las piezas de madera, antes de aplicar el acabado final. Es importante mencionar que cualquier imperfección que no se pueda eliminar con el lijado, cuando se aplique el acabado el defecto se acentuará. De ahí la importancia de conocer los defectos que pueden aparecer en la superficie lijada y cómo eliminar parte de éstos.

En la fabricación de lijas se utilizan diferentes tipos de abrasivos que en orden de su resistencia al desgaste son: esmeril, cuarzo, granate, óxido de aluminio y carburo de silicio. Los materiales que mejores resultados ofrecen para lijar la madera son el granate y el óxido de aluminio. La graduación del grano de las lijas para el lijado de la madera, deberá de variar de acuerdo con los defectos que tenga la superficie que se desea someter al proceso de lijado. La graduación que se emplea para denominar a cada una de las calidades de lija, se refiere a la cantidad de perforaciones que tiene una malla por pulgada cuadrada, a través de la cuál se hace pasar el abrasivo para ser depositado en la base de la lija.

Se hicieron pruebas con tres diferentes graduaciones de lija en cada una de las cinco probetas por especie probada, usando las lijas de grano 60, 80 y 100 . Con cada uno de los tres tamaños de grano, se rebajó aproximadamente $1 \mathrm{~mm}$ de espesor a las probetas, aplicando una fuerza manual de manera constante en el apoyo de la lijadora y en forma uniforme a través de toda la superficie. Para esta operación se utilizó una lijadora de banda de 6" de ancho con una longitud de 6.0 metros y con una velocidad lineal de 20 metros/segundo (Fig. 5). El abrasivo de la lija seleccionada fue el óxido de aluminio sobre respaldo de tela. El apoyo de la lijadora para ejercer la presión de la lija sobre la madera, fue de $15 \times 30 \mathrm{~cm}$ con un respaldo de lona.

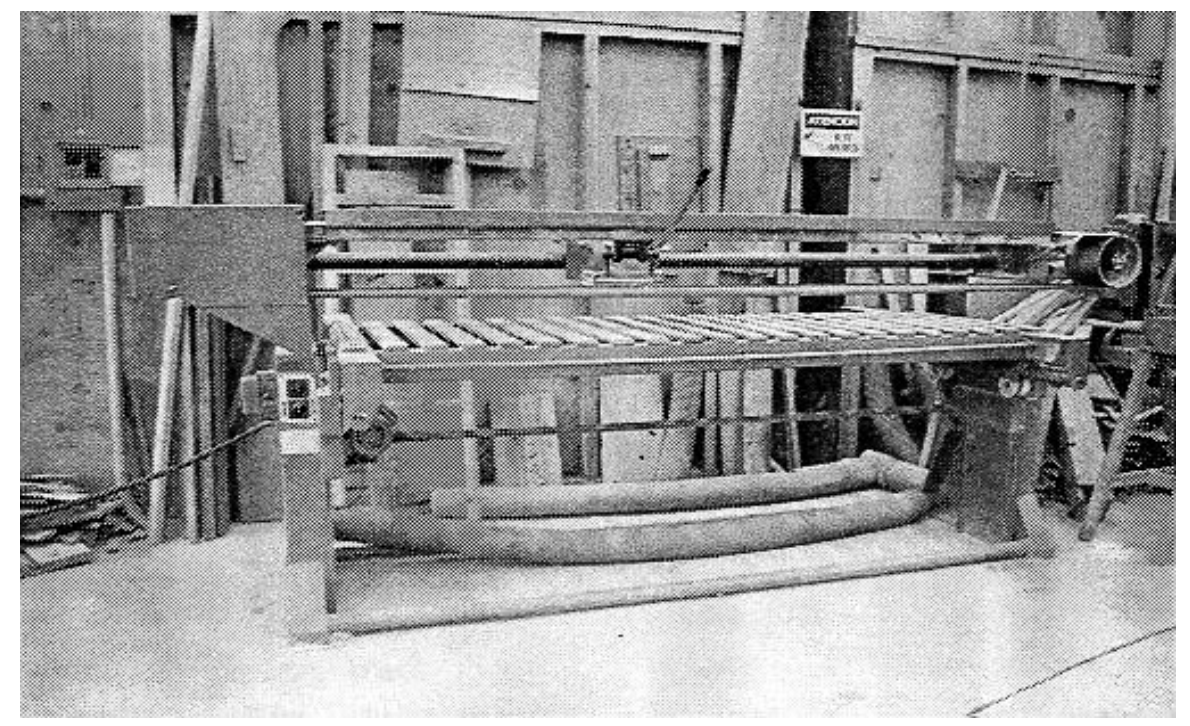

Figura 5. Máquina lijadora 
Antes de llevar a cabo las pruebas de lijado con cada uno de los diferentes tamaños de grano, se realizaron ensayos con la finalidad de probar el correcto funcionamiento del equipo. Ya con la maquinaria en condiciones apropiadas, se procedió a colocar la primera numeración de lija principiando por la graduación 60 . Las probetas se transportaron del cuarto para control de temperatura y humedad hacia la máquina lijadora dentro de bolsas de polietileno, para minimizar los cambios de contenido de humedad de la madera. El orden que se respetó para pasar cada una de las especies de madera, fue principiar con la especie de menor densidad relativa (0.19), para continuar así hasta la especie con mayor densidad relativa (0.90).

Los criterios para evaluar la superficie lijada de cada probeta fueron, las superficies rayadas y piezas con grano algodonoso (Davis, 1962) Cada uno de estos defectos se dividió en cinco posibles calificaciones: EXCELENTE (1), BUENO (2), REGULAR (3), POBRE (4) y MUY POBRE (5), dando así una calificación del 1 al 5 a cada probeta. La calificación se realizó en forma visual y se anotó en una hoja de registro que se elaboró para este fin. Cuando la evaluación final de las probetas sometidas a un lijado con la graduación de lija 60 de dió por terminada, procediendo a colocar la lija de graduación 80 , para repetir el procedimiento que se utilizó con el primer tipo de lija, y de igual forma se realizaron las pruebas con la lija de graduación 100, dando así por terminada la etapa de pruebas.

\section{RESULTADOS}

\section{Cepillado}

Con las calificaciones obtenidas se procedió a analizar los valores registrados en las hojas de resultados de cada especie. El primer paso fue obtener el porcentaje de piezas libres de defectos por especie, considerando para este fin únicamente a las tablas calificadas con los valores de Excelente (1) y Bueno (2). Con base en los datos obtenidos, a cada especie se le dió una calificación general de acuerdo con la Tabla 2.

Tabla 2. Rangos de clasificación final (Echenique y Plumptre, 1990)

\begin{tabular}{||l|c||}
\hline CALIFICACION & $\begin{array}{c}\% \text { PIEZAS LIBRES } \\
\text { DE DEFECTOS }\end{array}$ \\
\hline Excelente (1) & $90-100$ \\
Bueno (2) & $70-90$ \\
Regular (3) & $50-70$ \\
Pobre (4) & $30-50$ \\
Muy Pobre (5) & $0-30$ \\
\hline
\end{tabular}

Con base en esta clasificación se procedió a elaborar un listado de calificación final para cada especie probada, obteniéndose los resultados mostrados en la Tabla 3, correspondiente a las calificaciones para el ángulo de corte de $30^{\circ}$ en donde se puede observar que la densidad relativa no muestra una relación directa con la calidad de la superficie maquinada, pues en las especies calificadas con acabado excelente se observan rangos de densidad relativa entre 0.24 y 0.90 .

En la Tabla 4 se muestra la calificación de las especies sometidas a un cepillado con un ángulo de corte de $25^{\circ}$ en las cuchillas. En este listado la relación que podría existir entre la densidad relativa y la calidad del cepillado tampoco es notoria. Los resultados de las especies probadas con ángulo de corte de $20^{\circ}$ se muestran en la Tabla 5 donde se observa que la mayoría de las especies probadas tienen una calidad de superficie aceptable, notándose una ligera tendencia a tener mejor calidad en especies con mayor 
Tabla 3. Resultados de cepillado con un ángulo de corte en la cuchilla de 30/

\begin{tabular}{|c|c|}
\hline ESPECIE & $\begin{array}{c}\text { DR } \\
\text { (pa/vv) }\end{array}$ \\
\hline \multicolumn{2}{|l|}{ EXCELENTE } \\
\hline $\begin{array}{l}\text { Manilkara zapota } \\
\text { Platimiscium yucatanum } \\
\text { Lucuma salicifolia } \\
\text { Cupania sp } \\
\text { Fraxinus udhei } \\
\text { Guarea grandifolia } \\
\text { Pinus teocote } \\
\text { Liquidambar macrophylla } \\
\text { Casuarina cunninghamiana } \\
\text { Sw ietenia macrophylla } \\
\text { Persea americana } \\
\text { Talauma mexicana } \\
\text { Bursera simarouba } \\
\text { Pseudobombax ellipticum }\end{array}$ & $\begin{array}{l}0.90 \\
0.74 \\
0.71 \\
0.59 \\
0.53 \\
0.51 \\
0.49 \\
0.48 \\
0.42 \\
0.42 \\
0.40 \\
0.34 \\
0.30 \\
0.24\end{array}$ \\
\hline \multicolumn{2}{|l|}{ BUENO } \\
\hline $\begin{array}{l}\text { Lonchocarpus castilloi } \\
\text { Licania platypus } \\
\text { Astronium graveolens } \\
\text { Quercus sartorii } \\
\text { Magnolia schideana } \\
\text { Pinus patula } \\
\text { Vatairea lundellii } \\
\text { Acer skutchii } \\
\text { Pinus pseudostrobus } \\
\text { Schizolobium parahybum } \\
\text { Cedrela odorata }\end{array}$ & $\begin{array}{l}0.71 \\
0.61 \\
0.61 \\
0.53 \\
0.52 \\
0.51 \\
0.51 \\
0.40 \\
0.35 \\
0.35 \\
0.33\end{array}$ \\
\hline \multicolumn{2}{|l|}{ REGULAR } \\
\hline $\begin{array}{l}\text { Mirandaceltis monoica } \\
\text { Ceiba pentandra }\end{array}$ & $\begin{array}{l}0.65 \\
0.19\end{array}$ \\
\hline \multicolumn{2}{|l|}{ POBRE } \\
\hline $\begin{array}{l}\text { Ampelocera hottlei } \\
\text { Lysiloma bahamensis }\end{array}$ & $\begin{array}{l}0.65 \\
0.62\end{array}$ \\
\hline \multicolumn{2}{|l|}{ MUY POBRE } \\
\hline $\begin{array}{l}\text { Dialium guianense } \\
\text { Quercus crassifolia } \\
\text { Lysiloma acapulcensis } \\
\text { Vochysia guatemalensis }\end{array}$ & $\begin{array}{l}0.73 \\
0.68 \\
0.49 \\
0.42\end{array}$ \\
\hline
\end{tabular}

Tabla 4. Resultados de cepillado con un ángulo de corte en la cuchilla de 25/

\begin{tabular}{|c|c|}
\hline ESPECIE & $\begin{array}{c}\text { DR } \\
(\mathrm{pa} / \mathrm{vv})\end{array}$ \\
\hline \multicolumn{2}{|l|}{ EXCELENTE } \\
\hline $\begin{array}{l}\text { Manilkara zapota } \\
\text { Lucuma salicifolia } \\
\text { Quercus crassifolia } \\
\text { Astronium graveolens } \\
\text { Fraxinus uhdei } \\
\text { Vatairea lundellii } \\
\text { Guarea grandifolia } \\
\text { Pinus patula } \\
\text { Pinus teocote } \\
\text { Swietenia macrophylla } \\
\text { Acer skutchii } \\
\text { Pseudobombax ellipticum }\end{array}$ & $\begin{array}{l}0.90 \\
0.71 \\
0.68 \\
0.61 \\
0.53 \\
0.51 \\
0.51 \\
0.51 \\
0.49 \\
0.42 \\
0.40 \\
0.24\end{array}$ \\
\hline \multicolumn{2}{|l|}{ BUENO } \\
\hline $\begin{array}{l}\text { Platimiscium yucatanum } \\
\text { Lonchocarpus castilloi } \\
\text { Mirandaceltis monoica } \\
\text { Licania platypus } \\
\text { Quercus sartorii } \\
\text { Magnolia schideana } \\
\text { Casuarina cunninghamiana } \\
\text { Schizolobium parahybum } \\
\text { Talauma mexicana }\end{array}$ & $\begin{array}{l}0.74 \\
0.71 \\
0.65 \\
0.61 \\
0.53 \\
0.52 \\
0.42 \\
0.35 \\
0.34\end{array}$ \\
\hline \multicolumn{2}{|l|}{ REGULAR } \\
\hline $\begin{array}{l}\text { Cupania sp. } \\
\text { Liquidambar macrophylla } \\
\text { Persea americana }\end{array}$ & $\begin{array}{l}0.59 \\
0.48 \\
0.40\end{array}$ \\
\hline \multicolumn{2}{|l|}{ POBRE } \\
\hline $\begin{array}{l}\text { A mpelocera hottlei } \\
\text { Cedrela odorata } \\
\text { Bursera simarouba } \\
\text { Ceiba pentandra }\end{array}$ & $\begin{array}{l}0.65 \\
0.33 \\
0.30 \\
0.19\end{array}$ \\
\hline \multicolumn{2}{|l|}{ MUY POBRE } \\
\hline $\begin{array}{l}\text { Dialium guianense } \\
\text { Lysiloma bahamensis } \\
\text { Lysiloma acapulcensis } \\
\text { Vochysia guatemalensis } \\
\text { Pinus pseudostrobus }\end{array}$ & $\begin{array}{l}0.73 \\
0.62 \\
0.49 \\
0.44 \\
0.35\end{array}$ \\
\hline
\end{tabular}


Tabla 5. Resultados de cepillado con un ángulo de corte en la cuchilla de 20/

\begin{tabular}{|c|c|}
\hline ESPECIE & $\begin{array}{c}\mathrm{DR} \\
(\mathrm{pa} / \mathrm{vv})\end{array}$ \\
\hline \multicolumn{2}{|l|}{ EXCELENTE } \\
\hline $\begin{array}{l}\text { Manilkara zapota } \\
\text { Lucuma salicifolia } \\
\text { Astronium graveolens } \\
\text { Fraxinus uhdei } \\
\text { Magnolia schideana } \\
\text { Pinus patula } \\
\text { Guarea grandifolia } \\
\text { Liquidambar macrophylla } \\
\text { Sw ietenia macrophylla } \\
\text { Casuarina cunninghamiana } \\
\text { Acer skutchii } \\
\text { Talauma mexicana } \\
\text { Pseudobombax ellipticum }\end{array}$ & $\begin{array}{l}0.90 \\
0.71 \\
0.61 \\
0.53 \\
0.52 \\
0.51 \\
0.51 \\
0.48 \\
0.42 \\
0.40 \\
0.34 \\
0.24\end{array}$ \\
\hline \multicolumn{2}{|l|}{ BUENO } \\
\hline $\begin{array}{l}\text { Licania platypus } \\
\text { Cupania sp } \\
\text { Quercus sartorii } \\
\text { Pinus teocote } \\
\text { Vochysia guatemalensis } \\
\text { Persea americana } \\
\text { Schizolobium parahybum } \\
\text { Cedrela odorata } \\
\text { Ceiba pentandra }\end{array}$ & $\begin{array}{l}0.61 \\
0.59 \\
0.53 \\
0.49 \\
0.44 \\
0.40 \\
0.35 \\
0.33 \\
0.19\end{array}$ \\
\hline \multicolumn{2}{|l|}{ REGULAR } \\
\hline $\begin{array}{l}\text { Lysiloma acapulcensis } \\
\text { Bursera simarouba }\end{array}$ & $\begin{array}{l}0.49 \\
0.30\end{array}$ \\
\hline \multicolumn{2}{|l|}{ POBRE } \\
\hline $\begin{array}{l}\text { Platimiscium yucatanum } \\
\text { Lonchocarpus castilloi } \\
\text { Mirandaceltis monoica } \\
\text { Vatairea lundellii } \\
\text { Pinus pseudostrobus }\end{array}$ & $\begin{array}{l}0.74 \\
0.71 \\
0.65 \\
0.51 \\
0.35\end{array}$ \\
\hline \multicolumn{2}{|l|}{ MUY POBRE } \\
\hline $\begin{array}{l}\text { Dialium guianense } \\
\text { Quercus crassifolia } \\
\text { Ampelocera hottlei } \\
\text { Lysiloma bahamensis }\end{array}$ & $\begin{array}{l}0.73 \\
0.68 \\
0.65 \\
0.62\end{array}$ \\
\hline
\end{tabular}

densidad aunque existen algunas excepciones con especies de baja densidad y calificación de excelente.

\section{Discusión}

En esta prueba el comportamiento de las especies con respecto a cada uno de los cuatro defectos evaluados fue diferente en el mismo ángulo de corte probado, obteniendo algunas especies mejores resultados que otras. En las gráficas de resultados globales no se puede observar una relación muy directa entre densidad y porcentaje de piezas sin defectos. La figura 6 , para el ángulo de corte de 25/, es un caso típico de los resultados obtenidos. Las gráficas para los otros ángulos de corte son similares. Cuando se analizó cada defecto por separado se observó que en la mayoría de los casos esta relación sí se presenta. Por lo que respecta al grano levantado, el defecto fue más notorio en el ángulo de $25^{\circ}$, siendo más marcado en algunas especies superiores a 0.45 de densidad; en el ángulo de $20^{\circ}$ fue menos marcado y se presentó con mayor intensidad en algunas especies con densidad menor de 0.40 , mientras que con el ángulo de $30^{\circ}$ sólo se presentó en pocas especies, por lo que se concluye que para el defecto de grano levantado los mejores resultados se encontraron con un ángulo de corte de $30^{\circ}$ (Fig. 7).

El defecto del grano algodonoso se presentó en mayor número en las probetas sometidas a cepillado con ángulo de corte de $20^{\circ}$; este defecto disminuyó en las piezas probadas con un ángulo de corte de $25^{\circ}$ y los mejores resultados se obtuvieron con las especies probadas con un ángulo de corte de $30^{\circ}$ (Fig. 8).

Las marcas de viruta fueron más notorias en las piezas probadas con un ángulo de corte de $30^{\circ}$, se presentaron con menor intensidad con el ángulo de corte de $20^{\circ}$ y los mejores resultados se apreciaron en las piezas probadas con un ángulo 
modificado a $25^{\circ}$ (Fig. 9).

El defecto denominado grano arrancado es el que aparece con mayor frecuencia en los tres ángulos probados y es el que en forma general contribuyó con mayor peso a la clasificación de todas las especies probadas, este defecto aparece en mayor número con el ángulo de $25^{\circ}$, enseguida aparece con el ángulo de corte de $20^{\circ}$, y los mejores resultados se apreciaron con el ángulo de corte de $30^{\circ}$ (Fig. 10). En general los resultados más aceptable se observaron con el ángulo de corte de $30^{\circ}$, pues tres de los cuatro defectos evaluados fueron menores con este ángulo de corte.

\section{Lijado}

De igual manera como se procedió con las pruebas de cepillado con respecto a las calificaciones de la superficie de lijado para obtener el número de piezas libres de defectos, se consideraron las probetas con valores de 1 y 2, como Excelentes y Buenas. Con el porcentaje de piezas de piezas libres de defectos, se utilizó la Tabla 2 , para obtener una clasificación final de cada especie que permitiera elaborar tablas de resultados finales en cada una de las graduaciones de lijas probadas. Para el grado 60 se generó la Tabla 6 , para el grado 80 la Tabla 7 y la Tabla 8 para las piezas probadas con el grado 100.

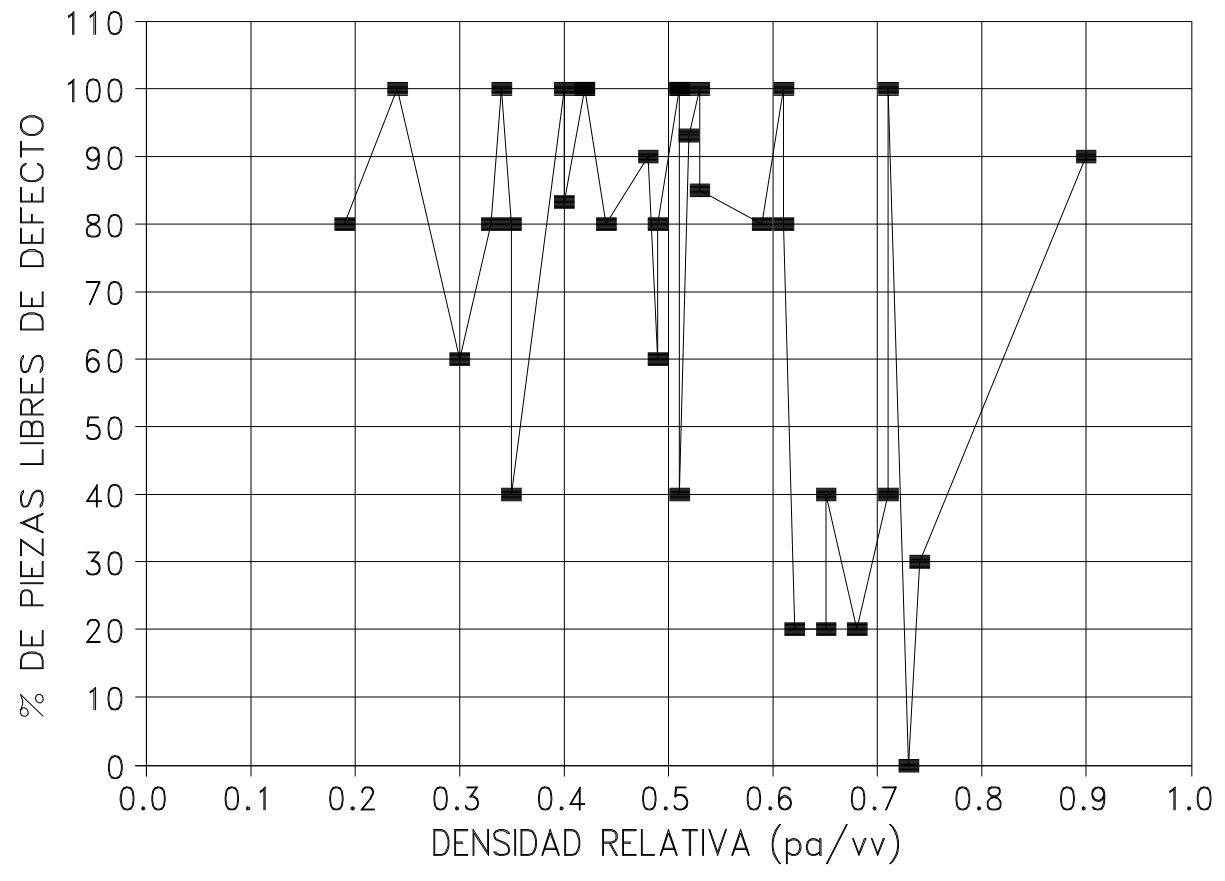

Figura 6. Gráfica de densidad relativa contra porcentajes de piezas sin defectos para la prueba de cepillado con un ángulo de corte de 25/ 


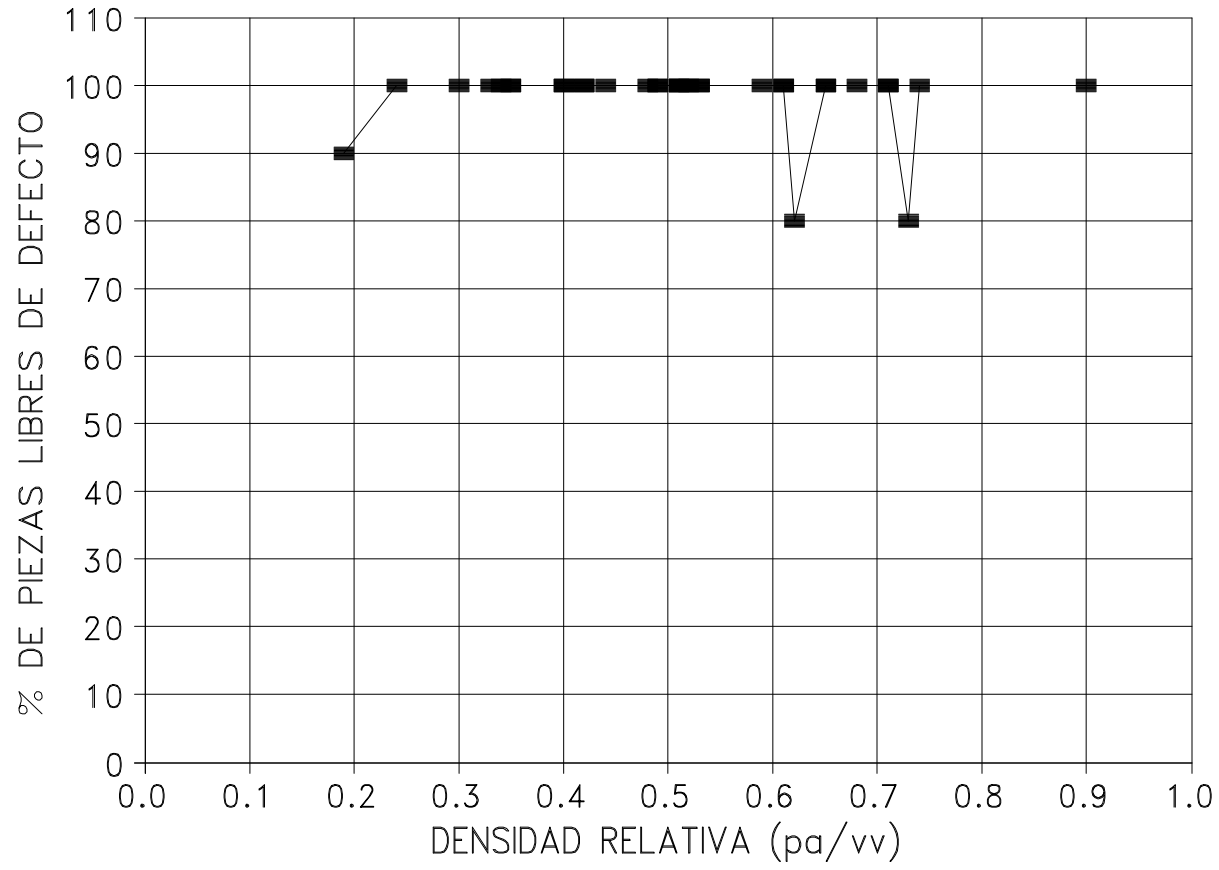

Figura 7. Gráfica de densidad relativa contra porcentajes de piezas sin defectos tomando en cuenta el grano levantado con ángulo de corte de 30/

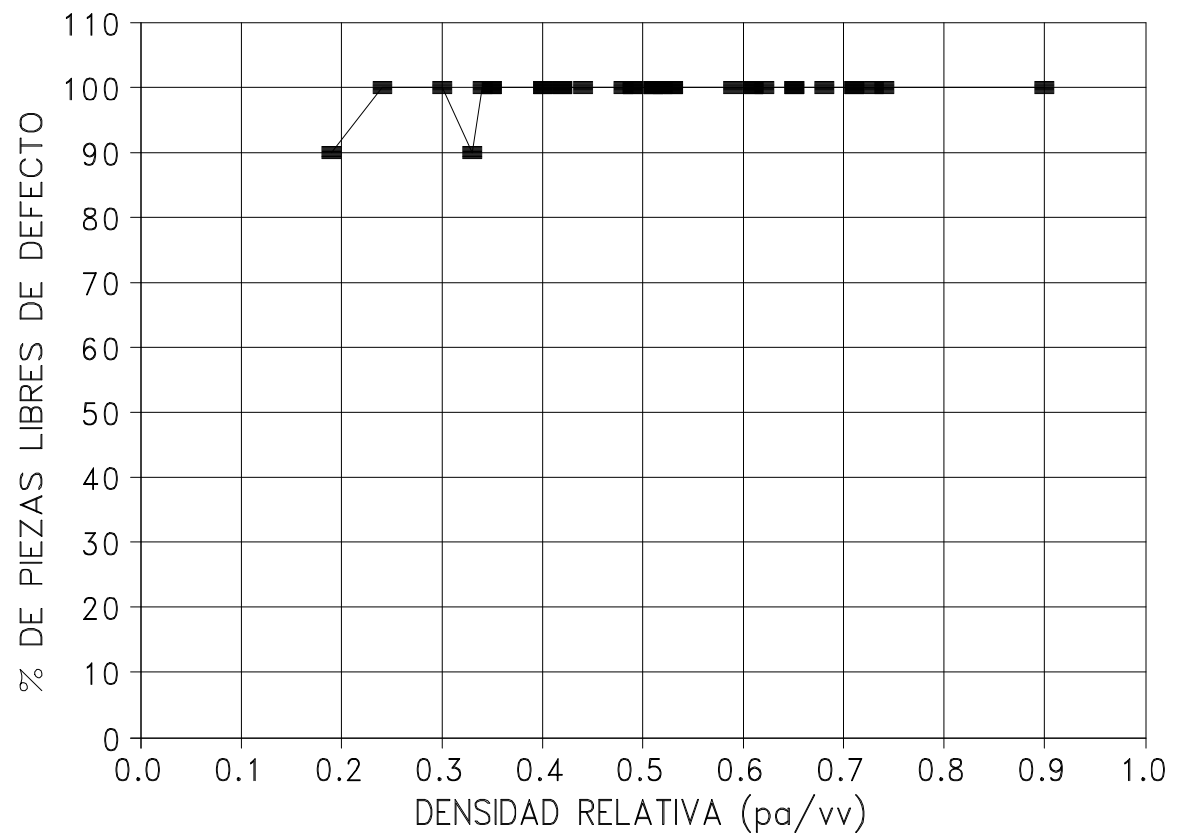

Figura 8. Gráfica de densidad relativa contra porcentajes de piezas sin defecto tomando en cuenta el grano algodonoso y ángulo de corte de 30/ 


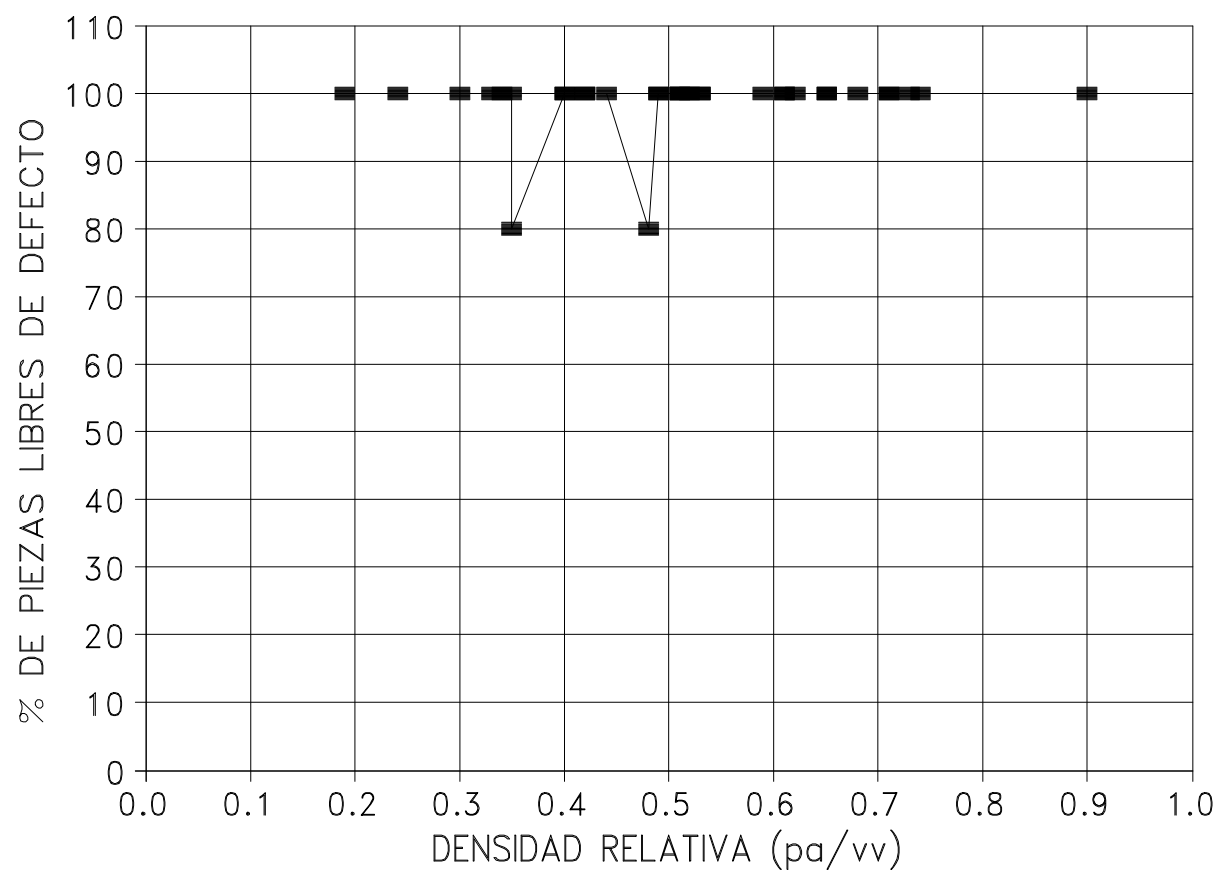

Figura 9. Gráfica de densidad relativa contra porcentaje de piezas sin defecto, tomando en cuenta marcas de viruta y ángulo de corte de 25/

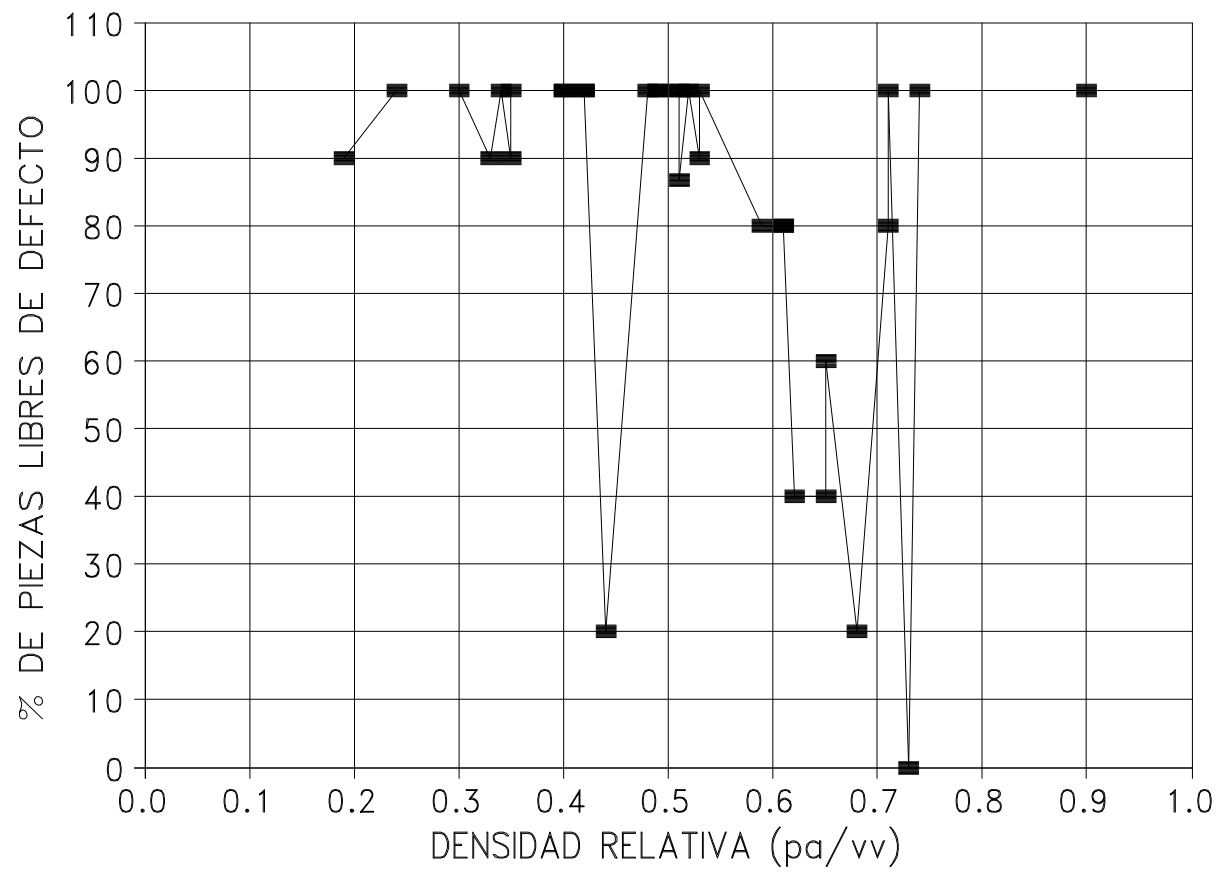

Figura 10. Gráfica de densidad relativa contra porcentajes de piezas sin defectos, tomando en cuenta el grano arrancado y ángulo de corte de 30/ 
Tabla 6. Resultados de lijado usando lija grano 60

\begin{tabular}{|c|c|}
\hline ESPECIE & $\begin{array}{c}\mathrm{DR} \\
(\mathrm{pa} / \mathrm{vv})\end{array}$ \\
\hline \multicolumn{2}{|l|}{ EXCELENTE } \\
\hline $\begin{array}{l}\text { Manilkara zapota } \\
\text { Platimiscium yucatanum } \\
\text { Lucuma salicifolia }\end{array}$ & $\begin{array}{l}0.90 \\
0.73 \\
0.71\end{array}$ \\
\hline \multicolumn{2}{|l|}{ REGULAR } \\
\hline $\begin{array}{l}\text { Dialium guianense } \\
\text { Fraxinus uhdei }\end{array}$ & $\begin{array}{l}0.73 \\
0.53\end{array}$ \\
\hline \multicolumn{2}{|l|}{ POBRE } \\
\hline $\begin{array}{l}\text { Lysiloma bahamensis } \\
\text { Licania platypus }\end{array}$ & $\begin{array}{l}0.62 \\
0.61\end{array}$ \\
\hline \multicolumn{2}{|l|}{ MUY POBRE } \\
\hline $\begin{array}{l}\text { Lonchocarpus castilloi } \\
\text { Quercus crassifolia } \\
\text { Mirandaceltis monoica } \\
\text { Ampelocera hottlei } \\
\text { Astronium graveolens } \\
\text { Cupania spp. } \\
\text { Quercus sartorii } \\
\text { Magnolia schiedeana } \\
\text { Vatairea lundellii } \\
\text { Guarea grandifolia } \\
\text { Pinus patula } \\
\text { Pinus teocote } \\
\text { Lysiloma acapulcensis } \\
\text { Liquidambar macrophylla } \\
\text { Vochysia guatemalensis } \\
\text { Sw ietenia macrophylla } \\
\text { Casuarina cunninghamiana } \\
\text { Acer skutchii } \\
\text { Persea americana } \\
\text { Pinus pseudostrobus } \\
\text { Schizolobium parahybum } \\
\text { Talauma mexicana } \\
\text { Cedrela odorata } \\
\text { Bursera simaruba } \\
\text { Pseudobombax ellipticum } \\
\text { Ceiba pentandra }\end{array}$ & $\begin{array}{l}0.71 \\
0.68 \\
0.65 \\
0.64 \\
0.61 \\
0.59 \\
0.53 \\
0.52 \\
0.51 \\
0.51 \\
0.51 \\
0.49 \\
0.49 \\
0.48 \\
0.44 \\
0.42 \\
0.42 \\
0.40 \\
0.40 \\
0.35 \\
0.35 \\
0.34 \\
0.33 \\
0.30 \\
0.24 \\
0.19\end{array}$ \\
\hline
\end{tabular}

Tabla 7. Resultados de lijado usando lija grano 80

\begin{tabular}{|c|c|}
\hline ESPECIES & $\begin{array}{c}\mathrm{DR} \\
(\mathrm{pa} / \mathrm{vv})\end{array}$ \\
\hline \multicolumn{2}{|l|}{ EXCELENTE } \\
\hline $\begin{array}{l}\text { Manilkara zapota } \\
\text { Dialium guianense } \\
\text { Lucuma salicifolia } \\
\text { Quercus crassifolia } \\
\text { Vatairea lundellii }\end{array}$ & $\begin{array}{l}0.90 \\
0.73 \\
0.71 \\
0.68 \\
0.51\end{array}$ \\
\hline \multicolumn{2}{|l|}{ BUENO } \\
\hline $\begin{array}{l}\text { Platimiscium yucatanum } \\
\text { Lysiloma bahamensis }\end{array}$ & $\begin{array}{l}0.73 \\
0.62\end{array}$ \\
\hline \multicolumn{2}{|l|}{ REGULAR } \\
\hline $\begin{array}{l}\text { Astronium graveolens } \\
\text { Fraxinus uhdei } \\
\text { Talauma mexicana }\end{array}$ & $\begin{array}{l}0.61 \\
0.53 \\
0.34\end{array}$ \\
\hline \multicolumn{2}{|l|}{ POBRE } \\
\hline Pinus patula & 0.51 \\
\hline \multicolumn{2}{|l|}{ MUY POBRE } \\
\hline $\begin{array}{l}\text { Lonchocarpus castilloi } \\
\text { Mirandacettis monoica } \\
\text { Ampelocera hottlei } \\
\text { Licania platypus } \\
\text { Cupania sp } \\
\text { Quercus sartorii } \\
\text { Magnolia schideana } \\
\text { Guarea grandifolia } \\
\text { Pinus teocote } \\
\text { Lysiloma acapulcensis } \\
\text { Liquidambar macrophylla } \\
\text { Vochysia guatemalensis } \\
\text { Sw ietenia macrophylla } \\
\text { Casuarina cunninghamiana } \\
\text { Acer skutchii } \\
\text { Persea americana } \\
\text { Pinus pseudostrobus } \\
\text { Schizolobium parahybum } \\
\text { Cedrela odorata } \\
\text { Bursera simarouba } \\
\text { Pseudobombax ellipticum } \\
\text { Ceiba pentandra }\end{array}$ & $\begin{array}{l}0.71 \\
0.65 \\
0.64 \\
0.61 \\
0.59 \\
0.53 \\
0.52 \\
0.51 \\
0.49 \\
0.49 \\
0.48 \\
0.44 \\
0.42 \\
0.42 \\
0.40 \\
0.40 \\
0.35 \\
0.35 \\
0.33 \\
0.30 \\
0.24 \\
0.19\end{array}$ \\
\hline
\end{tabular}


Tabla 8. Resultados de lijado usando lija grano 100

\begin{tabular}{|c|c|}
\hline ESPECIE & $\begin{array}{c}\mathrm{DR} \\
(\mathrm{pa} / \mathrm{vv})\end{array}$ \\
\hline \multicolumn{2}{|l|}{ EXCELENTE } \\
\hline $\begin{array}{l}\text { Manilkara zapota } \\
\text { Dialium guianense } \\
\text { Platimiscium yucatanum } \\
\text { Lucuma salicifolia } \\
\text { Lonchocarpus castilloi } \\
\text { Quercus crassifolia } \\
\text { Mirandaceltis monoica } \\
\text { Ampelocera hottlei } \\
\text { Lysiloma bahamensis } \\
\text { Astronium graveolens } \\
\text { Licania platypus } \\
\text { Cupania spp. } \\
\text { Quercus sartorii } \\
\text { Fraxinus uhdei } \\
\text { Magnolia schideana } \\
\text { Vatairea lundellii } \\
\text { Guarea grandifolia } \\
\text { Pinus patula } \\
\text { Pinus teocote } \\
\text { Lysiloma acapulcensis } \\
\text { Liquidambar macrophylla } \\
\text { Vochysia guatemalensis } \\
\text { Casuarina cunninghamiana } \\
\text { Acer skutchii } \\
\text { Persea americana } \\
\text { Pinus pseudostrobus } \\
\text { Schizolobium parahybum } \\
\text { Talauma mexicana } \\
\text { Cedrela odorata }\end{array}$ & $\begin{array}{l}0.90 \\
0.73 \\
0.73 \\
0.71 \\
0.71 \\
0.68 \\
0.65 \\
0.64 \\
0.62 \\
0.61 \\
0.61 \\
0.59 \\
0.53 \\
0.53 \\
0.52 \\
0.51 \\
0.51 \\
0.51 \\
0.49 \\
0.49 \\
0.48 \\
0.44 \\
0.42 \\
0.40 \\
0.40 \\
0.35 \\
0.35 \\
0.34 \\
0.33\end{array}$ \\
\hline \multicolumn{2}{|l|}{ BUENO } \\
\hline $\begin{array}{l}\text { Sw ietenia macrophylla } \\
\text { Pseudobombax ellipticum }\end{array}$ & $\begin{array}{l}0.42 \\
0.24\end{array}$ \\
\hline \multicolumn{2}{|l|}{ REGULAR } \\
\hline Bursera simarouba & 0.30 \\
\hline \multicolumn{2}{|l|}{ MUY POBRE } \\
\hline Ceiba pentandra & 0.19 \\
\hline
\end{tabular}

\section{Discusión}

Los defectos que aparecen en esta operación son: piezas rayadas y piezas con superficie algodonosa. En la prueba con graduación 60 y 80 , la superficie rayada se presentó principalmente en las probetas con densidad relativa baja, disminuyendo en forma proporcional con el aumento de la densidad, como ejemplo se incluye la Figura 11 para las pruebas realizadas con lija de graduación 80.

El defecto de rayado en la madera se presenta en todas las superficies de cualquier especie con menor o mayor profundidad dependiendo de la graduación de la lija, tendiendo a minimizarse con lijas de mayor graduación. Para poder desaparecer cualquier defecto de la superficie, como huellas de cepillado, cortes de sierra, o alguno de los defectos antes mencionados, se deberá empezar a lijar con una lija de grano relativamente grueso en una primera pasada y posteriormente ir disminuyendo el número de grano de la lija, pasando a uno más fino.

El grano algodonoso se presentó en la mayoría de las probetas tanto las de densidad baja como las de alta, dentro de las pruebas realizadas con graduación de lija 60 y 80, pero en las probetas sometidas a la graduación de lija 100 apareció en pequeño porcentaje, por lo tanto, el defecto de la superficie algodonosa se puede eliminar con el aumento de la finura del grano de la lija.

En las piezas probadas, los mejores resultados se obtuvieron usando lija con un grado de 100. Se observó que, con lija de graduación 80 , los resultados en cuanto a calidad de la superficie fueron intermedios y finalmente los resultados con menor calidad aparecieron con el uso de lija con graduación de 60 . 


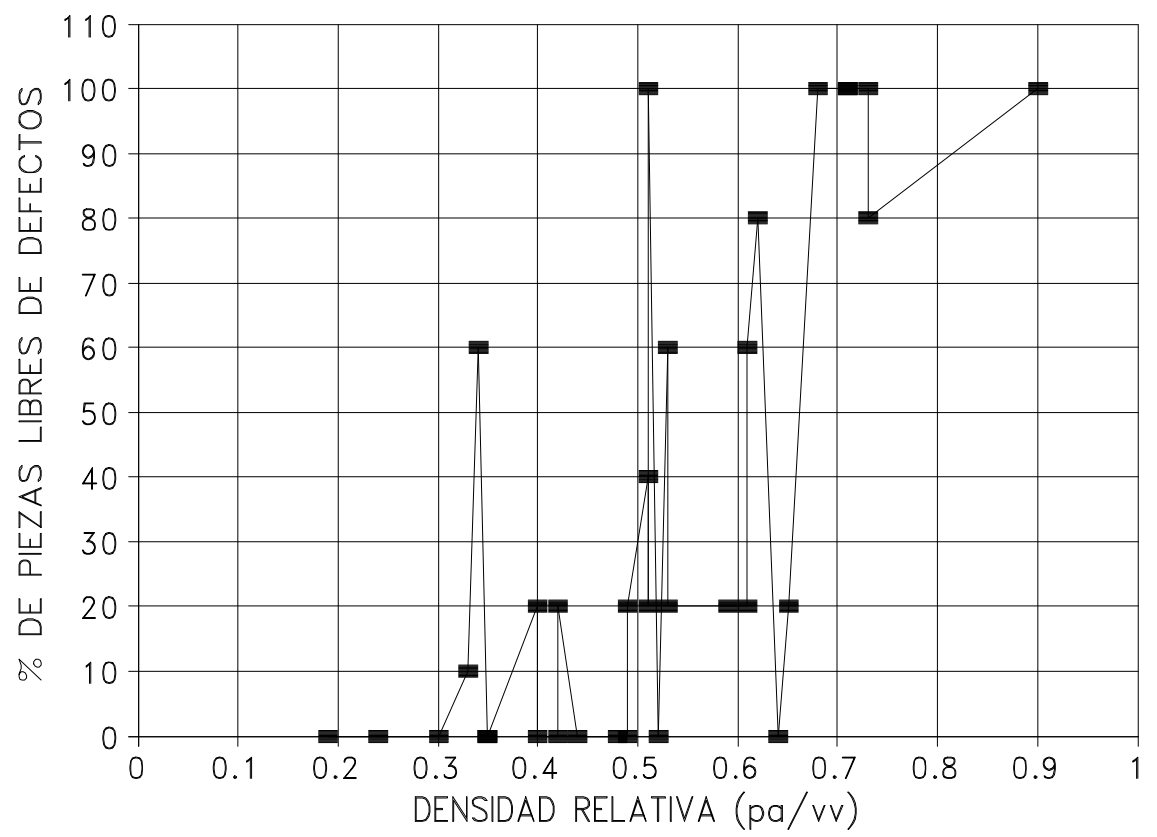

Figura 11. Gráfica de densidad relativa contra porcentajes de piezas sin defectos en la prueba de lijado con granulación 80

\section{CONCLUSIONES}

Con la información presentada en las tablas y gráficas en este trabajo se puede inferir la calidad que, en general, tendrá cada especie al ser sometida a los dos procesos estudiados. En la Tabla 9 se presentan las especies divididas en categorías para el proceso de cepillado. Doce de las especies tuvieron resultados de Excelente, nueve se pueden considerar como Buenas, cinco caen en la categoría de Regular, cinco pertenecen al grupo de Pobre y las restantes se pueden considerar Muy Pobres.

La Tabla 10 presenta el mismo resúmen relacionado con la prueba de lijado. En este caso, únicamente tres especies se pueden considerar como Excelentes, con los tres tipos de lija empleados, cinco especies se pueden incluir en la categoría de Buenas y la gran mayoría se agrupan en las categorías de Regular a Muy Pobres. La razón de esta discrepancia es que con las lijas de grado 60 y 80 casi todas las especies se consideran en la categoría más baja o Muy Pobre, al registrar rayado severo. Es el caso opuesto al de la lija grado 100, para la cual casi todas las especies se pueden considerar como Excelentes.

\section{RECONOCIMIENTOS}

Este proyecto se realizó con recursos fiscales asignados al Departamento de Productos Forestales y Conservación de Bosques, con la clave 902-13. Agradecemos los comentarios críticos del M. en I. V. Rubén Ordóñez Candelaria y del Dr. Raymundo Dávalos Sotelo para la presentación final de los resultados y en particular de las conclusiones. 
Tabla 9. Clasificación final para cepillado incluyendo resultados de los tres ángulos de corte

\begin{tabular}{|c|c|}
\hline$\overline{\text { ESPECIE }}$ & $\begin{array}{c}\mathrm{DR} \\
(\mathrm{pa} / \mathrm{vv})\end{array}$ \\
\hline \multicolumn{2}{|l|}{ EXCELENTE } \\
\hline $\begin{array}{l}\text { Manilkara zapota } \\
\text { Lucuma salicifolia } \\
\text { Astronium graveolens } \\
\text { Fraxinus uhdei } \\
\text { Guarea grandifolia } \\
\text { Pinus patula } \\
\text { Pinus teocote } \\
\text { Sw ietenia macrophylla } \\
\text { Casuarina cunninghamiana } \\
\text { Acer skutchii } \\
\text { Talauma mexicana } \\
\text { Pseudobombax ellipticum }\end{array}$ & $\begin{array}{l}0.90 \\
0.71 \\
0.61 \\
0.53 \\
0.51 \\
0.51 \\
0.49 \\
0.42 \\
0.42 \\
0.40 \\
0.34 \\
0.24\end{array}$ \\
\hline \multicolumn{2}{|l|}{ BUENO } \\
\hline $\begin{array}{l}\text { Platimiscium yucatanum } \\
\text { Licania platypus } \\
\text { Cupania sp } \\
\text { Quercus sartorii } \\
\text { Magnolia schideana } \\
\text { Vatairea lundellii } \\
\text { Liquidambar macrophylla } \\
\text { Persea americana } \\
\text { Schizolobium parahybum }\end{array}$ & $\begin{array}{l}0.73 \\
0.61 \\
0.59 \\
0.53 \\
0.52 \\
0.51 \\
0.48 \\
0.40 \\
0.35\end{array}$ \\
\hline \multicolumn{2}{|l|}{ REGULAR } \\
\hline $\begin{array}{l}\text { Lonchocarpus castilloi } \\
\text { Mirandaceltis monoica } \\
\text { Cedrela odorata } \\
\text { Bursera simarouba } \\
\text { Ceiba pentandra }\end{array}$ & $\begin{array}{l}0.71 \\
0.65 \\
0.33 \\
0.30 \\
0.19\end{array}$ \\
\hline \multicolumn{2}{|l|}{ POBRE } \\
\hline $\begin{array}{l}\text { Ampelocera hottlei } \\
\text { Quercus crassifolia } \\
\text { Lysiloma acapulcensis } \\
\text { Vochysia guatemalensis } \\
\text { Pinus pseudostrobus }\end{array}$ & $\begin{array}{l}0.64 \\
0.68 \\
0.49 \\
0.44 \\
0.35\end{array}$ \\
\hline \multicolumn{2}{|l|}{ MUY POBRE } \\
\hline $\begin{array}{l}\text { Dialium guianense } \\
\text { Lysiloma bahamensis }\end{array}$ & $\begin{array}{l}0.73 \\
0.62\end{array}$ \\
\hline
\end{tabular}

Tabla 10. Clasificación final para lijado con las tres clases de lija

\begin{tabular}{|c|c|}
\hline ESPECIE & $\begin{array}{c}\text { DR } \\
(\mathrm{pa} / \mathrm{vv})\end{array}$ \\
\hline \multicolumn{2}{|l|}{ EXCELENTE } \\
\hline $\begin{array}{l}\text { Manilkara zapota } \\
\text { Platimiscium yucatanum } \\
\text { Lucuma salicifolia }\end{array}$ & $\begin{array}{l}0.90 \\
0.73 \\
0.71\end{array}$ \\
\hline \multicolumn{2}{|l|}{ BUENO } \\
\hline $\begin{array}{l}\text { Dialium guianense } \\
\text { Quercus crassifolia } \\
\text { Lysiloma bahamensis } \\
\text { Fraxinus uhdei } \\
\text { Vatairea lundellii }\end{array}$ & $\begin{array}{l}0.73 \\
0.68 \\
0.62 \\
0.53 \\
0.51\end{array}$ \\
\hline \multicolumn{2}{|l|}{ REGULAR } \\
\hline $\begin{array}{l}\text { Licania platypus } \\
\text { Astronium graveolens } \\
\text { Pinus patula } \\
\text { Talauma mexicana }\end{array}$ & $\begin{array}{l}0.61 \\
0.61 \\
0.51 \\
0.34\end{array}$ \\
\hline \multicolumn{2}{|l|}{ POBRE } \\
\hline $\begin{array}{l}\text { Lonchocarpus castilloi } \\
\text { Mirandacetis monoica } \\
\text { Ampelocera hottlei } \\
\text { Cupania sp } \\
\text { Quercus sartorii } \\
\text { Magnolia schideana } \\
\text { Guarea grandifolia } \\
\text { Pinus teocote } \\
\text { Lysiloma acapulcensis } \\
\text { Liquidambar macrophylla } \\
\text { Vochysia guatemalensis } \\
\text { Casuarina cunninghamiana } \\
\text { Swietenia macrophylla } \\
\text { Acer skutchii } \\
\text { Persea americana } \\
\text { Pinus pseudostrobus } \\
\text { Schizolobium parahybum } \\
\text { Cedrela odorata } \\
\text { Bursera simarouba } \\
\text { Pseudobombax ellipticum }\end{array}$ & $\begin{array}{l}0.71 \\
0.65 \\
0.64 \\
0.59 \\
0.53 \\
0.52 \\
0.51 \\
0.49 \\
0.49 \\
0.48 \\
0.44 \\
0.42 \\
0.42 \\
0.40 \\
0.40 \\
0.35 \\
0.35 \\
0.33 \\
0.30 \\
0.24\end{array}$ \\
\hline \multicolumn{2}{|l|}{ MUY POBRE } \\
\hline Ceiba pentandra & 0.19 \\
\hline
\end{tabular}




\section{REFERENCIAS}

American Society for Testing and Materials. 1993. Standard methods of conducting machining tests of wood and wood based materials. D-1666-64. p:348-373.

Cantin, M. 1965. The machining properties of 16 Eastern Canadian woods. Canadian Department of Forestry. Publ. 1111. $27 \mathrm{p}$.

Davis, E. 1962. Machining and related characteristics of United States hardwoods. U.S. Department of Agriculture. Forest Service. 49 p.
Echenique M., R. y R.A. Plumptre. 1990. A Guide to the use of Mexican and Belizean timbers. Tropical Forestry Papers 20, Oxford Forestry Institute, Department of Plant Sciences, University of Oxford, Oxford, Inglaterra.

Longwood, F. 1961. Puerto Rican woods. Their machining, seasoning and related characteristics. Agriculture handbook No. 205. U.S. Department of Agriculture. Forest Service. 34 p. 